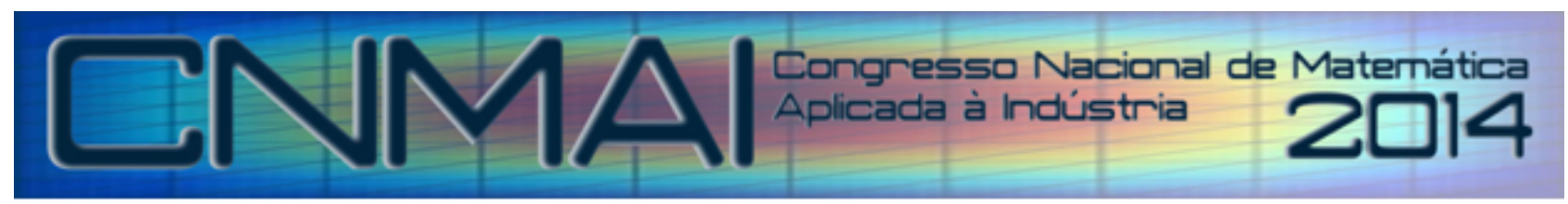

18 a 21 de novembro de 2014, Caldas Novas - Goiás

\title{
Dimensionamento de lotes em fundições de pequeno porte com múltiplos fornos CNMAI2014-0114
}

\author{
Franklina M. B. Toledo, fran@icmc. usp. br ${ }^{1}$ \\ Victor C. B. Camargo, victor. camargo@producao. uftm. edu . br ${ }^{2}$ \\ Nathan P. B. Oliveira, nathanpartel@gmail. com ${ }^{1}$ \\ Viviane S. Tonaki, vtonaki@yahoo.com.br ${ }^{1}$ \\ ${ }^{1}$ Universidade de São Paulo - Instituto de Ciências Matemáticas e de Computação \\ Av. Trabalhado São-carlense, 400, 13.560-970, São Carlos-SP, Brasil
}

${ }^{2}$ Universidade Federal do Triângulo Mineiro - Departamento de Engenharia de Produção
Av. Doutor Randolfo Borges Jr, 1250, Univerdecidade - 38.064-200, Uberaba-MG, Brasil

Resumo. O planejamento da produção em fundições de pequeno porte é especialmente difícil devido à variedade de ligas e itens que são produzidos. Este problema pode ser resumido a determinar quais itens serão produzidos em cada período do horizonte de planejamento e, consequentemente, quais ligas devem ser fundidas para garantir essa produção. Essas decisões estão diretamente relacionadas, pois cada item é vazado a partir de uma liga específica. Logo, os itens a serem produzidos num dado período dependem das ligas que forem fundidas. Em cada período, o número de fusões é limitado e o volume de liga fundida correspondente à capacidade do forno utilizado. Na literatura, esse problema considerando apenas um forno para a produção foi tratado utilizando métodos exatos e heurísticos. Embora na prática seja relevante considerar no processo de planejamento mais de um tipo de forno, poucos trabalhos incluem esta questão. Planejar a produção considerando vários fornos é especialmente importante por questões de economia de energia elétrica e, consequentemente, redução dos custos de produção. Neste trabalho, propomos um modelo inteiro misto para o problema de dimensionamento de lotes inspirado numa fundição de pequeno porte do Estado de São Paulo. A fundição dispõe de dois fornos que não podem ser utilizados simultaneamente. Testes computacionais foram conduzidos com o objetivo de comparar o planejamento integrado dos fornos com soluções da literatura para problemas em que apenas um forno é tratado.

Palavras-chave: planejamento de produção; otimização inteira; fundição; múltiplos fornos

\section{INTRODUÇÃO}

Neste trabalho, abordamos o problema de planejamento de produção em fundições de pequeno porte. De forma geral, um plano de produção deve especificar quais ligas serão fundidas e quais peças serão vazadas a partir destas ligas. A definição de um plano de produção eficiente é particularmente difícil devido à grande variedade de ligas e itens que são produzidos.

As fundições de pequeno porte têm um importante papel na economia brasileira, pois pertencem a cadeia produtiva de indústrias de diferentes setores, como, por exemplo, automobilístico e agrícola. Além disso, são responsáveis por muitos empregos diretos e indiretos.

Devido a sua importância econômica e ao desafio científico que o problema de planejamento de sua produção representa, vários autores estudaram o problema. Stawowy e Duda (2012) apresentam uma revisão de artigos que abordam o problema. Em particular, Araujo et al. (2008), Teixeira.Jr. et al. (2010), Tonaki e Toledo (2010), Camargo et al. (2012a), 
Duda e Stawowy (2013) propuseram métodos e/ou modelos para o problema de planejamento da produção em fundições de pequeno porte.

Embora seja relevante para algumas fundições considerar no planejamento da produção a utilização não simultânea de fornos diferentes, poucos trabalhos tratam esta questão. Silva e Morabito (2004) propuseram uma heurística construtiva gulosa para o problema em que vários fornos estão disponíveis. Os autores modelaram o problema como um problema de corte de estoque unidimensional em que os objetos a serem cortados são barras que representam a capacidade de cada um dos fornos (em quilos) e os itens a serem produzidos são representados por peças de diferentes tamanhos (em quilos). $\mathrm{O}$ objetivo do problema é maximizar a utilização semanal dos fornos, ou seja, o número de itens produzidos. Uma instância real do problema foi resolvida pela heurística, resultando numa produtividade maior que a solução utilizada na prática.

Recentemente, Navarenho e Camargo (2014) propuseram um novo modelo para o problema com múltiplos fornos. Um modelo para o planejamento da produção de dois estágios e múltiplas máquinas no segundo estágio proposto em (Camargo et al., 2012b) foi adaptado para representar o ambiente produtivo de uma fundição com múltiplos fornos de uso não simultâneo. O objetivo é minimizar os estoques e os atrasos na produção dos itens. Testes computacionais mostraram que a utilização de vários fornos torna o planejamento mais flexível e possibilita a minimização dos custos de produção.

Neste trabalho, propomos um modelo inteiro misto para o problema de planejamento de produção com múltiplos fornos que foi inspirado numa fundição de pequeno porte do Estado de São Paulo. A fundição dispõe de dois fornos que não podem ser utilizados simultaneamente. Testes computacionais foram conduzidos com o objetivo de comparar o planejamento integrado dos fornos com soluções da literatura para problemas em que apenas um forno é tratado. Também comparamos os resultados com uma simulação da política de planejamento adotada pela empresa.

$\mathrm{O}$ artigo está organizado da seguinte forma. Primeiramente, definimos o problema estudado e apresentamos o modelo proposto. Em seguida, reportamos os testes computacionais realizados para validação do modelo. Finalmente, as considerações finais sobre o trabalho são apresentadas.

\section{DEFINIÇÃO DO PROBLEMA E O MODELO PROPOSTO}

A produção de uma fundição de mercado atende a uma carteira de clientes que, em geral, é bastante vasta e diversa. Logo, o planejador da produção trabalha com muitos pedidos que podem conter diferentes itens, distintos quanto a liga em que são fundidos e a quantidade em que são demandados. Estas características do planejamento da produção o tornam especialmente difícil e, muitas vezes, demorado de ser realizado.

Tipicamente, os fornos e as máquinas de moldagem são os principais equipamentos de uma fundição de mercado de pequeno porte. Seu processo de produção pode ser resumido nas seguintes etapas: a) moldagem; b) elaboração e fusão das ligas; e c) fabricação dos itens (vazamento dos moldes e tratamento dos itens).

O gargalo de produção do problema estudado é a fusão das ligas, ou seja, a utilização dos fornos disponíveis. Para a fundição em que este estudo foi inspirado, existem dois fornos disponíveis que, por questões técnicas, não podem ser ligados simultaneamente. Os fornos têm capacidade máxima de fusão e, embora não exista um mínimo, quando operam abaixo de sua capacidade seu desgaste é mais significativo, podendo requerer manutenções mais frequentes. Como os fornos têm capacidades significativamente distintas (um deles é em torno de três vezes maior que o outro), um plano de produção que considere alternativas no uso dos fornos, leva à economia de custos de manutenção e de operação da fundição.

O planejamento da produção em fundições de pequeno porte pode ser caracterizado como um processo de dois estágios, em que no primeiro estágio são definidas as ligas a serem fundidas e no segundo os itens que serão produzidos a partir destas ligas. Estas decisões estão diretamente relacionadas, pois cada item é vazado a partir de uma liga específica. Logo, os itens a serem produzidos num dado período dependem das ligas fundidas. Em cada período, o número de fusões é limitado e o volume de liga fundida correspondente à capacidade do forno utilizado.

Tendo como base o trabalho de (Araujo, 2003), propomos um modelo que considera múltiplos fornos. Os ínidces, parâmetros e variáveis utilizados no modelo são descritos nas Tab. 1 e Tab. 2.

Tabela 1. Índices do modelo.

\begin{tabular}{ll}
\hline Índices & \\
\hline$k \in\{1, \ldots, K\}=\mathcal{K}$ & conjunto de ligas; \\
$i \in\{1, \ldots, N\}=\mathcal{N}$ & conjunto de itens; \\
$f \in\{1, \ldots, F\}=\mathcal{F}$ & conjunto de fornos; \\
$t \in\{1, \ldots, T\}=\mathcal{T}$ & conjunto de períodos; \\
$\eta \in\left\{1, \ldots, U_{T}\right\}=\mathcal{L}$ & conjunto de subperíodos. \\
\hline
\end{tabular}


Tabela 2. Parâmetros e variáveis do modelo.

\begin{tabular}{ll}
\hline Parâmetros & \\
\hline$u_{f}$ & tempo de fusão do forno $f$ (em horas); \\
$\operatorname{cap}_{f}$ & capacidade de fusão do forno $f$ (em quilos); \\
$\exp$ & capacidade de fusão em cada período do horizonte de planejamento (em tempo); \\
$M a x_{t}$ & número máximo de subperíodos em um período do horizonte de planejamento \\
& $\left(M a x_{t}=\left\lfloor\exp / \min \left(u_{f}\right)\right\rfloor\right) ;$ \\
$P_{t}$ & primeiro subperíodo do período $t, P_{t}=1+(t-1) * M a x_{t} ;$ \\
$U_{t}$ & último subperíodo do período $t, U_{t}=t * M a x_{t} ;$ \\
$\mathcal{L}_{t}=\left\{P_{t}, \ldots, U_{t}\right\}$ & conjunto de subperíodos do período $t ;$ \\
$S(k)$ & conjunto de itens que são fabricados com a liga $k$ \\
$b_{i t}$ & penalidade pelo atraso em atender a demanda do item $i$ no período $t ;$ \\
$h_{i t}$ & penalidade pelo estoque do item $i$ no final do período $t ;$ \\
$d_{i t}$ & demanda do item $i$ no período $t ;$ \\
$\rho_{i}$ & peso do item $i$ (em quilos). \\
\hline Variáveis & \\
\hline$B_{i t}$ & quantidade do item $i$ em atraso no final do período $t ;$ \\
$I_{i t}$ & quantidade do item $i$ em estoque no final do período $t ;$ \\
$X_{i \eta f}$ & quantidade do item $i$ produzida no subperíodo $\eta$ no forno $f ;$ \\
$L_{k \eta f}$ & variável binária que assume o valor 1 se a liga $k$ for fundida no subperíodo $\eta$ no forno $f$, \\
& zero caso contrário.
\end{tabular}

$$
\begin{aligned}
& \operatorname{Min} \sum_{t \in \mathcal{T}} \sum_{i \in \mathcal{N}}\left(b_{i t} \cdot B_{i t}+h_{i t} \cdot I_{i t}\right) \\
& \text { s.a } \\
& I_{i, t-1}+\sum_{\eta \in \mathcal{L}_{t}} \sum_{f \in \mathcal{F}} X_{i \eta f}+B_{i t} \\
& =d_{i t}+I_{i t}+B_{i, t-1} \quad i \in \mathcal{N} ; t \in \mathcal{T} \\
& \sum_{i \in S(k)} \rho_{i} \cdot X_{i \eta f} \leq \operatorname{cap}_{f} \cdot L_{k \eta f} \quad k \in \mathcal{K} ; \eta \in \mathcal{L} ; f \in \mathcal{F} \\
& \sum_{f \in \mathcal{F}} \sum_{k \in \mathcal{K}} \sum_{\eta \in \mathcal{L}_{t}} u_{f} \cdot L_{k \eta f} \leq \exp \quad t \in \mathcal{T} \\
& \sum_{k \in \mathcal{K}} \sum_{f \in \mathcal{F}} L_{k \eta f} \leq 1 \quad \eta \in \mathcal{L} \\
& I_{i 0}=0, B_{i 0}=0 \\
& X_{i \eta f} \in \mathcal{Z}_{+} \\
& i \in \mathcal{N} ; \eta \in \mathcal{L} ; f \in \mathcal{F} \\
& I_{i t}, B_{i t} \geq 0 \\
& i \in \mathcal{N} ; t \in \mathcal{T} \\
& L_{k \eta f} \in\{0,1\} \\
& k \in \mathcal{K} ; \eta \in \mathcal{L} ; f \in \mathcal{F}
\end{aligned}
$$

A função objetivo (1) visa minimizar a soma das penalidades por atraso e por antecipação (estoque). Nas restrições (2) garantimos o atendimento da demanda. O limite de capacidade dos fornos é imposto pelas restrições (3), enquanto o número de fornadas por período é limitado em (4). Em cada um dos subperíodos, apenas uma liga pode ser fundida em um único forno (5). Sem perda de generalidade os estoques iniciais e os itens em atraso no início do horizonte são considerados nulos em (6). O domínio das variáveis é descrito nas restrições (7)-(9).

Para tratar a possibilidade de fusão em múltiplos fornos, adaptamos as variáveis de produção $\left(X_{i \eta f}\right.$ e $\left.L_{k \eta f}\right)$ e as restrições do modelo de Araujo (2003) para considerar os vários fornos. Além disso, incluímos no modelo um novo conjunto de restrições. As restrições (4) garantem que a capacidade de fusão da fundição em horas é limitada a seu expediente, e não ao número de vezes que um dado forno pode ser utilizado. Como os fornos têm tamanhos diferentes e o tempo necessário para a fusão de cada um deles é distinto, o número de fusões em um período é variável, diferente do modelo de Araujo (2003) em que esse número é pré-fixado. Para tratar esta incerteza, um limite máximo de fusões por dia é determinado por $\operatorname{Max}_{t}$. 


\section{RESULTADOS COMPUTACIONAIS}

Para avaliar o modelo proposto foram utilizados nove exemplos gerados com base em carteiras reais de uma fundição de pequeno porte do Estado de São Paulo. Os exemplos foram resolvidos considerando os dois fornos da fundição, um com capacidade máxima de $140 \mathrm{~kg}$ e o outro com capacidade máxima de $380 \mathrm{~kg}$. O tempo de fusão dos fornos é, respectivamente, igual a 1 e a 0,8 unidades de tempo do expediente, que tem um total de 10 unidades. Os demais dados dos exemplos são resumidos na Tab. 3.

Tabela 3. Dados dos exemplos.

\begin{tabular}{c|rrr}
\hline Exemplo & Itens & Ligas & Períodos \\
\hline 1 & 398 & 37 & 5 \\
2 & 26 & 11 & 1 \\
3 & 40 & 15 & 2 \\
4 & 111 & 14 & 3 \\
5 & 29 & 11 & 2 \\
6 & 168 & 27 & 5 \\
7 & 109 & 29 & 5 \\
8 & 90 & 20 & 4 \\
9 & 114 & 21 & 5 \\
\hline
\end{tabular}

O modelo proposto foi escrito em linguagem de modelagem e resolvido utilizando o software de otimização CPLEX versão 12.5 (configuração padrão) num servidor HP Xeon E5-2620 2.0 GHz 64Gb RAM com sistema operacional Ubuntu 12.04. Os testes foram realizados com limite de tempo (TL) de uma hora para cada exemplo.

Os resultados obtidos para os 9 exemplos são resumidos na Tab. 4. A primeira coluna da tabela indica o exemplo que foi resolvido. Nas três colunas seguintes, reportamos o custo da melhor solução obtida (FO), o desvio (Desvio (\%)), o tempo total de resolução (T(s)) e a perda de material em quilos (Perda $(\mathrm{Kg})$ ), ou seja, a soma da subutilização dos fornos. O desvio é dado por:

$$
\text { Desvio }=100 \cdot \frac{F O-L I}{L I}
$$

em que: LI é o limitante inferior do problema.

Nas colunas 6-9, apresentamos os resultados obtidos pelo software de otimização CPLEX versão 12.5 (configuração padrão) resolvendo os exemplos considerando apenas um forno como proposto em (Araujo, 2003). Neste caso, assumimos que apenas o forno maior está disponível. Destacamos que, como os autores consideram na função objetivo custos para a troca de ligas durante o planejamento da produção, sua função objetivo foi substituída pela função objetivo (1) do modelo aqui proposto para comparações de resultados. Em negrito destacamos os melhores resultados obtidos. Na última coluna da tabela, apontamos a economia percentual obtida utilizando o modelo com múltiplos fornos, ou seja:

$$
\text { Economia }=100 \cdot \frac{F O_{1 \text { forno }}-F O_{2 \text { fornos }}}{F O_{1 \text { forno }}} .
$$

\begin{tabular}{|c|c|c|c|c|c|c|c|c|c|}
\hline \multirow[t]{2}{*}{ Ex. } & \multicolumn{4}{|c|}{ Dois Fornos } & \multicolumn{4}{|c|}{ Um único forno } & \multirow{2}{*}{$\begin{array}{r}\text { Economia } \\
(\%)\end{array}$} \\
\hline & F.O. & Desvio (\%) & $\mathrm{T}(\mathrm{s})$ & Perda $(\mathrm{Kg})$ & F.O. & Desvio (\%) & $\mathrm{T}(\mathrm{s})$ & Perda (Kg) & \\
\hline 1 & 4.140 .830 & 0,4 & TL & 15 & 4.137.259 & 0,3 & TL & 10 & $-0,09$ \\
\hline 2 & $\mathbf{0}$ & 0,0 & 0 & 1.045 & 68 & 0,0 & 1 & 2.413 & - \\
\hline 3 & 28.333 & 0,0 & 1 & 1.367 & 28.474 & 0,0 & 3 & 3.067 & 0,50 \\
\hline 4 & 59.159 & 5,2 & TL & 1.618 & 59.381 & 0,8 & $\mathrm{TL}$ & 2.564 & 0,37 \\
\hline 5 & 7.675 & 0,0 & 112 & 2.329 & 7.675 & 0,0 & 10 & 3.389 & 0,00 \\
\hline 6 & 834.871 & 3,0 & TL & 4.147 & 837.493 & 3,1 & TL & 6.591 & 0,31 \\
\hline 7 & 803.179 & 7,4 & $\mathrm{TL}$ & 2.997 & 820.403 & 6,3 & TL & 7.019 & 2,10 \\
\hline 8 & 707.385 & 2,2 & TL & 1.754 & 706.956 & 0,8 & TL & 3.423 & $-0,06$ \\
\hline 9 & 840.939 & 2,1 & $\mathrm{TL}$ & 2.323 & 841.239 & 2,1 & TL & 2.626 & 0,04 \\
\hline Média & & 2,3 & & 1.955 & & 1,5 & & 3.456 & 0,39 \\
\hline
\end{tabular}

Tabela 4. Análise do modelo proposto.

Como esperado, ao abordarmos o problema considerando os dois fornos disponíveis, os valores da função objetivo são inferiores aos valores do planejamento com um único forno, para a maioria das carteiras. Apenas para os Exemplos $1 \mathrm{e}$ 
8, os resultados são piores em menos de $0,1 \%$ e para o Exemplo 5 as duas abordagens levam a resultados iguais. Também observamos que, em média, o desvio da solução ótima para o problema com múltiplos fornos (2,3\%) é maior que o desvio para o problema com um único forno $(1,4 \%)$, o que pode levar a uma redução ainda maior nos valores da função objetivo. Para os exemplos em que o modelo para dois fornos apresentou melhoria nos valores da função objetivo, esta varia de 0,04\% (Exemplo 9) a 2,1\% (Exemplo 7). O Exemplo 2 não foi incluído na média, uma vez que o valor da função objetivo é zero quando vários fornos são considerados. Além disso, temos um melhor aproveitamento da capacidade dos fornos, ao utilizarmos os dois fornos, a subutilização é reduzida em média $43 \%$.

Também comparamos os resultados obtidos com uma simulação da política de planejamento utilizada pela fundição. $\mathrm{Na}$ Tab. 5, apresentamos os resultados obtidos. É importante destacar que a política da empresa considera em seu planejamento os dois fornos. Quando comparado à simulação da política de planejamento da empresa, os resultados obtidos pelo modelo são significativamente melhores, tanto em relação ao custo quanto em relação à subutilização dos fornos.

Tabela 5. Comparação com uma simulação da política de planejamento da fundição.

\begin{tabular}{c|rr|rr}
\hline Ex. & \multicolumn{2}{|c|}{ Modelo Proposto } & \multicolumn{2}{c}{ Simulação da Prática } \\
& F.O. & Perda $(\mathrm{Kg})$ & F.O. & Perda $(\mathrm{Kg})$ \\
\hline 1 & $\mathbf{4 . 1 4 0 . 8 3 0}$ & $\mathbf{1 5}$ & 4.630 .160 & 4.607 \\
2 & $\mathbf{0}$ & $\mathbf{1 . 0 4 5}$ & 68 & 2.413 \\
3 & $\mathbf{2 8 . 3 3 3}$ & $\mathbf{1 . 3 6 7}$ & 49.739 & 3.130 \\
4 & $\mathbf{5 9 . 1 5 9}$ & $\mathbf{1 . 6 1 8}$ & 91.506 & 3.390 \\
5 & $\mathbf{7 . 6 7 5}$ & $\mathbf{2 . 3 2 9}$ & 25.401 & 3.395 \\
6 & $\mathbf{8 3 4 . 8 7 1}$ & $\mathbf{4 . 1 4 7}$ & 1.242 .410 & 6.591 \\
7 & $\mathbf{8 0 3 . 1 7 9}$ & $\mathbf{2 . 9 9 7}$ & 1.313 .720 & 8.047 \\
8 & $\mathbf{7 0 7 . 3 8 5}$ & $\mathbf{1 . 7 5 4}$ & 1.006 .510 & 3.457 \\
9 & $\mathbf{8 4 0 . 9 3 9}$ & $\mathbf{2 . 3 2 3}$ & 1.034 .170 & 4.241 \\
\hline Média & \multicolumn{3}{|c}{} & 4.363 \\
\hline
\end{tabular}

\section{CONSIDERAÇÕES FINAIS}

O modelo proposto para o planejamento da produção de fundições de pequeno porte com múltiplos fornos se mostrou eficiente, resultando em planos de produção com custos melhores que o modelo que considera apenas um forno e também que a simulação da política de planejamento atualmente adotada pela empresa estudada. As soluções também são significativamente melhores com relação a utilização dos fornos.

Extensões do modelo ainda podem ser exploradas, considerando, por exemplo, penalidades para a troca de fornos. Além disso, buscar alternativas aos softwares comerciais de otimização para resolver o problema é muito importante, uma vez que os softwares comerciais têm custo relevante quando comparado ao orçamento de empresas de pequeno porte. Nesta direção, também seria interessante desenvolver métodos heurísticos independentes de softwares comerciais para este problema.

\section{AGRADECIMENTOS}

Os autores agradecem a FAPESP (2012/20278-0 e 2013/04505-0), ao CNPq (153155/2012-4 e 300713/2010-0), ao engenheiro e a programadora de produção da empresa.

\section{REFERÊNCIAS}

Araujo, S. A. 2003. Modelos e métodos para o planejamento e programação da produção aplicado no setor de fundições. Tese de Doutorado, Instituto de Ciências Matemáticas e de Computação - USP.

Araujo, S. A., Arenales, M. N., e Clark, A. R. 2008. Lot-sizing and furnace scheduling in small foundries. Computers \& Operations Research, 35, 916-932.

Camargo, V. C. B., Mattiolli, L., e Toledo, F. M. B. 2012a. A knapsack problem as a tool to solve the production planning problem in small foundries. Computers \& Operations Research, 39, 86 - 92.

Camargo, V. C. B., Toledo, F. M. B., e Almada-Lobo, B. 2012b. Three time-based scale formulations for the two-stage lot sizing and scheduling in process industries. Journal of the Operational Research Society, 63, 1613-1630.

Duda, J., e Stawowy, A. 2013. Optimization methods for lot-sizing problem in an automated foundry. Archives of Metallurgy and Materials, 58(3), 863-866.

Navarenho, D., e Camargo, V. C. B. 2014. Planejamento da produção de múltiplos fornos não-simultâneos em uma fundição de pequeno porte. Livro de resumos XVI Oficina Nacional de Problemas de Corte e Empacotamento, Planejamento e Programação da Produção e Correlatos, 1, 142. 
Silva, R. J., e Morabito, R. 2004. Otimização da programação de cargas de forno em uma fábrica de fundição em aço inox. Gestão \& Produção, 11, 135-151.

Stawowy, A., e Duda, J. 2012. Models and algorithms for production planning and scheduling in foundries - current state and development perspectives. Archives of Foundry Engineering, 12(2), 69-74.

Teixeira.Jr., R. F., Fernandes, F. C. F., e Pereira, N. A. 2010. Binary integer programming formulations for scheduling in market-driven foundries. Computers \& Industrial Engineering, 59, 425-435.

Tonaki, V. S., e Toledo, F. M. B. 2010. An approach to solve the lot-sizing and scheduling problem in market-driven foundries. Journal of the Operational Research Society, 61, 108 - 114.

\title{
RESPONSABILIDADE AUTORAL
}

“O(s) autor(es) é(são) o(s) único(s) responsável(is) pelo conteúdo deste trabalho”.

\section{Production planning for small-scale foundries with multiple furnaces codigo CNMAI2014-0114}

\author{
Franklina M. B. Toledo, fran@icmc. usp. br ${ }^{1}$ \\ Victor C. B. Camargo, victor. camargo@producao. uftm. edu . br ${ }^{2}$ \\ Nathan P. B. Oliveira, nathanpartel@gmail. com ${ }^{1}$ \\ Viviane S. Tonaki, vtonaki@yahoo.com.br ${ }^{1}$ \\ ${ }^{2}$ Universidade de São Paulo - Instituto de Ciências Matemáticas e de Computação \\ Av. Trabalhado São-carlense, 400, 13.560-970, São Carlos-SP, Brasil \\ ${ }^{3}$ Universidade Federal do Triângulo Mineiro - Departamento de Engenharia de Produção \\ Av. Doutor Randolfo Borges Jr, 1250, Univerdecidade - 38.064-200, Uberaba-MG, Brasil
}

\begin{abstract}
Production planning in small-scale foundries is especially challenging due to the wide variety of alloys used and items produced. This problem can be summarized by determining which items will be produced at each period of the planning horizon and, consequently which alloys should be forged to ensure production. These decisions are directly related as each item is forged from a specific alloy. Therefore, the items to be produced in a given period depend on the alloys which were forged. At each period, the number of times an alloy is forged is limited and the volume of the molten alloy corresponds to the capacity of the furnace used. In the literature, this problem considering only one furnace for production was addressed using exact methods and heuristics. Although in practice it would be relevant to consider more than one type of furnace, few papers include this issue. Planning the production for multiple furnaces is especially important when considering saving electricity, and consequently reducing production costs. In this paper, we propose a mixed integer model for production planning based on the activities of a small-scale foundry in the state of Sao Paulo. This foundry makes uses of two furnaces which cannot be used simultaneously. Computational tests were conducted with the aim of comparing the multiple furnace model with solutions from the literature concerning problems in which only one furnace is used.
\end{abstract}

Keywords: Production Planning, Integer Optimization, Foundry, Multiple Furnaces. 